EDITORIAL

\title{
Breaking Down Territorial Boundaries: A Call to All Professions and Disciplines
}

\author{
The Deputy Editor and the Editorial Board, Disaster Medicine and Public Health Preparedness
}

S table societies are positively influenced by appropriately designed and orchestrated support services, with contributions from and oversight by numerous professional disciplines. As societies mature, the society-supporting roles of these professional disciplines, which include politics, economics, security, law, and medicine, among others, become interlinked and interdependent. When called on to contribute to the resolution of a natural or manmade disaster, the professional disciplines are expected to share common goals to protect, maintain, and enhance a safe, healthy, and thriving community. The public health system is at the nexus of these disciplines and, by necessity, has had to expand its attention from its historical role of health and medical care to address communications, transportation, the judiciary, education, public safety, and many other elements of society that inextricably come together during times of crisis. ${ }^{1}$

Addressing these many challenges simultaneously involves the participation of many divergent groups who, in turn, are often required to depart from their traditional roles and instead cross and (at times) trespass on the professional boundaries of others. Such activities, altruistically aimed at efficiently recovering and rehabilitating a community and protecting its society, concomitantly introduce stress within the individual support groups and challenge their coordination with others. For these support groups to function optimally for the public good, they must plan and prepare for interactions with other complementary (and sometimes competing) groups before a disaster and on a continuing basis. Specifically, the public health system must continually communicate with other support groups to improve the functionality of the collective contributions. Unfortunately, such intertwined planning and communication exist only in a primitive state at best.

Disasters, regardless of their cause(s), are a great test of society, challenging the members of that society and the institutional structures that support them. Specifically, disasters reveal the insufficient bonds that bind support groups, causing each to function vertically rather than horizontally during periods of crisis, despite "peacetime" training to the contrary. This contributes to the societal vulnerability seen in disasters. As such, the most sensitive indicator of societal disruption is the functioning capacity of its public health system. ${ }^{2}$ Disaster response is an abrupt collision of multiple disciplines that includes both direct and indirect influences that range from clinical skill sets to public health information and security concerns.
Unfortunately, support groups frequently underappreciate the importance of this operational interdependency, and often operate independently (ie, in "silos") both before and during a disaster. Contributing to this lack of functionality with other groups is the false, typically untested assumption that robust horizontal communication and cooperation will somehow magically appear at the time of a disaster. Furthermore, disaster preparedness has suffered from a significant lack of common definitions for public health planning and response. In an attempt to introduce a broadly encompassing language, the term all-hazards has been adopted. As a reference for incident command structure this makes sense because the structure stays consistent regardless of the hazard. However, for public health preparedness planning and response, this adopted term tends to be confusing. Certainly, the planning and response to mass injury care, such as from a conventional bombing, is significantly different from mass illness management for an influenza pandemic.

These issues highlight why the American Medical Association journal Disaster of Medicine and Public Health Preparedness is so important and vital. The journal will promote communication across service and professional boundaries to expose those vulnerabilities that inherently require common solutions and strategies. As other medical and public health disciplines have evolved from a scientific base to create robust systems to better respond to public health burdens, such as trauma, cardiac disease, and recently cerebral vascular attacks, so too will disaster medicine. The journal will seek to become the evidence base for the discussion, development, and evolution of a nationwide disaster medical system, based firmly on the public health system. It will provide impetus for a public health emergency operations center to provide population-based decision making and triage. ${ }^{3}$ The journal will promote a common language and provide common definitions to better understand what exactly we are preparing for.

This journal will aim to educate all disciplines in protecting the public health during disasters and their aftermath. It will serve as a platform for discussing the roles that all contributors play in optimizing public health. The journal will also address ethical and legal issues surrounding standards of care and liability coverage for those medical and public health volunteers courageous enough to respond. This approach will examine the entire spectrum of challenges, from those dealing with local limitations 


\section{Editorial}

to those affected by the growing influence of globalization. This journal invites all disciplines to join us in this first step forward.

Received and accepted for publication March 29, 2007.

ISSN: 1935-7893 (C) 2007 by the American Medical Association and Lippincott Williams \& Wilkins.

DOI: 10.1097/DMP.0b013e3180654ca5

\section{REFERENCES}

1. Institute of Medicine. Report on Public Health in the 21st Century. Washington, DC: Institute of Medicine; 2002.

2. Burkle FM. Globalization and disasters: issues of public health, state capacity and political action. J Int Affairs. 2003;241-265.

3. Burkle FM, Jr. Population-based triage management in response to surgecapacity requirements during a large-scale bioevent disaster. Acad Emerg Med. 2006;13:1118-1129. 